\title{
Seguridad del neonato hospitalizado. Aproximaciones y propuestas.
}

\section{Safety of hospitalized neonate. Approximations and Proposals}

E.E.I. Lucrecia Guadalupe Sánchez Bañuelos,• Lic. Enf. Javier Pérez Gutiérrez•

E.E.P. Flor Elizabeth Tamariz Velázquez•• Mtra. Margarita Delgado Rubio ..•

\section{Resumen}

La seguridad del paciente forma parte de las prioridades de los sistemas de salud a nivel mundial. Es a través de la Alianza Mundial por la Seguridad de los Pacientes que la Organización Mundial de la Salud (OMS) planteó el objetivo de prevenir eventos adversos durante la atención de las personas en las instituciones de salud, asegurando así una atención libre de riesgos.

El individuo en su etapa de desarrollo neonatal, se encuentra más vulnerable a sufrir lesiones durante su estancia hospitalaria, por lo que existe la necesidad de contar con medidas de seguridad específicas para evitar riesgos durante su cuidado. Conforme a lo anterior se realizan propuestas de lineamientos aplicadas a este grupo etéreo, basadas en las seis Metas Internacionales para la Seguridad del Paciente establecidas por la Organización Mundial de la Salud (OMS).

En la búsqueda bibliográfica realizada en bases de datos como: Artemisa en línea, Pubmed, Scielo, Cochrane Plus, se identificaron algunos documentos que hacen referencia a estrategias de seguridad para pacientes neonatos; sin embargo es de importancia se realicen más investigaciones en enfermería para un cuidado seguro a este grupo de pacientes

"Alumnos de la Especialidad de Enfermería del Neonato del Instituto Nacional de Perinatología (INPer),

**Coordinadora de la Especialidad de Enfermería del Neonato del INPer,

***Jefa del Depto de Enseñanza de Enfermería del INPer

FeCha de ReCiBIDO: 30 ENERO 2012

Fecha de EnVIado: 21 DE Febrero 2012

FECHA DE ACEPTADO: 14 DE MARZO 2012

Clave:

Seguridad del Paciente, Eventos Adversos, Neonato, Enfermería, lineamientos. 


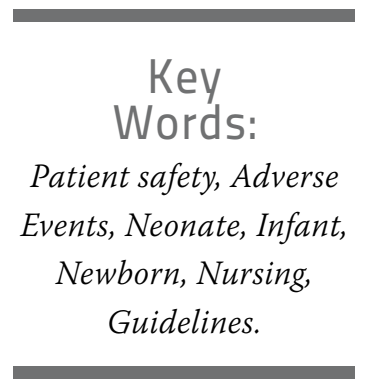

Abstract

Patient safety is a priority of health systems worldwide. Through the World Alliance for Patient Safety, the World Health Organization established the objective of preventing adverse events during the attention of persons in health institutions, thus assuring a risk free attention.

The individual in his/her neonatal development is more vulnerable to suffering lesions in his/her hospital stay, and because of this, there is a necessity of having specific safety measures to prevent risks during his/her care. As a result, guidelines proposals related to this group of persons are developed based on the six International Goals for the Patient Safety established by the World Health Organization (WHO)

In the literature search in databases such as Artemisa online, Pubmed, Scielo, Cochrane Plus, several documents referencing safety strategies for neonatal patients were identified; however, it is important to do more nursing research in order to achieve a safe care for this group of patients.

\section{INTRODUCCIÓN}

La Seguridad del paciente neonato requiere de estrategias específicas por las características propias de su etapa de desarrollo. La Organización Mundial de la Salud (OMS) en octubre de 2004 a través de la Alianza para la Seguridad del Paciente propuso coordinar, difundir y acelerar las mejoras en esta materia en todo el mundo. ${ }^{1}$

En los países desarrollados, se estima que hasta 1 de cada 10 pacientes hospitalizados sufren daños como resultado de la atención recibida; en países en vías de desarrollo la cifra es mayor. Lo anterior es importante pues en materia de seguridad evitar que los pacientes sufran daños durante el tratamiento $y$ atención es trascendental ${ }^{1}$ por lo que la prevención de los eventos adversos que pueden agregarse al estado de salud de los pacientes y generarle pérdidas económicas, daño psicosocial para el propio paciente y en algunos casos incluso para quien es el cuidador principal es una prioridad.

Específicamente los aspectos característicos así como la complejidad de las Unidades de Cuidados Intensivos Neonatales (UCIN) representan un medio ambiente distinto a otras áreas hospitalarias, lo que aunado a la vulnerabilidad de la población neonatal aumenta el riesgo de errores médicos. Derivado de lo anterior es conveniente contar con una visión general de los problemas de seguridad específicos para el cuidado intensivo neonatal además de identificar las estrategias para mejorar el proceso de atención siempre con la visión de una práctica segura. ${ }^{2}$

Actualmente la medición de la seguridad del paciente es necesaria para mejorar el cuidado dentro de estas unidades al comparar la atención prestada y determinar los cambios en respuesta a las intervenciones de seguridad o programas establecidos.
Bajo este marco, seguir las tendencias de seguridad establecidas en un servicio o unidad a través del tiempo es importante para identificar los eventos específicos; los métodos utilizados pueden incluir la presentación de informes, la observación directa, grabaciones en vídeo, gráficas de revisión, herramientas de activación y métodos automatizados de auditoría. ${ }^{3}$

El carácter de centro especializado en atención de embarazos de alto riesgo del Instituto Nacional de Perinatología en México, implica contar entre sus servicios con Unidades de Cuidados Intensivos Neonatales, por lo que es relevante considerar entre sus líneas de acción específicas las correspondientes con las Metas Internacionales de Seguridad del Paciente, en este caso para el neonato hospitalizado.

Con objeto de contribuir a mejorar la seguridad de los neonatos atendidos en esta institución, en 
este documento se plasman algunas de las estrategias y propuestas para tal fin.

\section{Desarrollo}

El Neonato se define como todo recién nacido desde el momento de nacer hasta los 28 días de vida. Se han propuesto numerosas clasificaciones para el recién nacido tomando en cuenta; el peso de nacimiento, la edad gestacional y el grado de crecimiento intrauterino, lo que ha permitido identificar grupos de neonatos con diferentes riesgos específicos de enfermedad, muerte y eventuales secuelas, lo que también obliga a establecer el tratamiento adecuado en forma oportuna y a determinar los recursos adicionales que se deben considerar.

La Organización Mundial de la Salud (oms), con el apoyo de centros europeos, fijó la división de prematurez en las 37 semanas, basado en que los recién nacidos entre 37-38 semanas serían de bajo riesgo, especialmente en países en desarrollo, donde este grupo se comporta como recién nacidos maduros y por tanto no necesitan atención especial. ${ }^{4}$

Hablar de seguridad en el paciente neonato implica, entre otras cosas, realizar un análisis y reflexión bioética que involucra a todos los profesionales que brindan la atención a fin de reconocer la importancia del reporte y notificación de eventos adversos. Derivado de lo anterior la OMS estableció, en su momento, como una de sus cuatro acciones iniciales formular una taxonomía de seguridad del paciente que sirviera para notificar sucesos adversos y elaborar sistemas de notificación y aprendizaje para facilitar el análisis de las causas que originan errores, prevenirlos y así evitar incidentes no deseados. ${ }^{5}$

Específicamente en las Unidades de Cuidados Intensivos Neonatales (UCIN) deben registrarse de manera multi-institucional los eventos adversos, esta información es sumamente importante por lo que se debe puntualizar; tipo de evento, etiología, evolución y mecanismos y/o medidas de prevención. Lo anterior facilitará el abordaje y análisis de sucesos que ocurrieron, esto sin dejar de reconocer que los efectos benéficos del reporte en esta área son aun difíciles de evaluar a partir de la evidencia disponible y por lo tanto aún falta seguir investigando. ${ }^{3}$

En un estudio piloto realizado por R. Ursprung y cols, se buscó determinar la viabilidad y la utilidad de la auditoría sobre seguridad en una Unidad de Cuidados Intensivos, se tenían como antecedentes la detección oportuna de errores, incluidos comentarios para el personal clínico en la mejora de seguridad centrada en el paciente. Se concluyó que las auditorías de rutina pueden detectar una amplia gama de errores y problemas importantes de seguridad, esto dio lugar a rápidos cambios en la política y la práctica. El personal aceptó el fomento de una cultura para el reporte de errores en beneficio de la seguridad del paciente. ${ }^{6}$

El sentirnos seguros es algo que busca el hombre durante toda su vida, luego de cubrir las necesidades fisiológicas, la seguridad está ubicada en el segundo escalón de la pirámide de Maslow. ${ }^{7}$ No obstante, el cuidado de la salud en los ámbitos hospitalarios y ambulatorios para el paciente neonato puede significar un riesgo a esta seguridad debido a la conjugación compleja de procesos tecnológicos e intervenciones humanas, que si bien contribuyen en acciones benéficas, también incluyen un abanico de posibilidades de práctica insegura para la ocurrencia de eventos adversos. ${ }^{8}$

Un evento adverso es considerado como el daño, lesión o incluso la muerte (evento centinela) causado por el tratamiento de una enfermedad o estado del paciente por los profesionales de salud, y que no obedece a la propia enfermedad o estados subyacentes. ${ }^{9}$

Con el propósito de minimizar la posibilidad de que se presente algún evento adverso durante la atención de los pacientes que acuden a los servicios médicos, la OMS estableció las siguientes estrategias:

1. Identificación correcta de los pacientes.

2. Mejora de la comunicación efectiva.

3. Mejora en la seguridad de los medicamentos de alto riesgo.

4. Garantizar cirugías con el lugar correcto, el procedimiento correcto y el paciente correcto.

5. Reducción de las infecciones adquiridas por la atención.

6. Reducción del riesgo de las lesiones del paciente por caídas. $^{1}$

En México existen dos instancias directamente involucradas con el reporte de errores ocurridos en los hospitales:

a) La Subsecretaría de Innovación

y Calidad que dictamina

el proceso de evaluación

para la acreditación de establecimientos del sistema de protección social en salud, vigilando que éstos cumplan con los requerimientos 
indispensables de capacidad, seguridad y calidad, en los procesos para la adecuada atención de los beneficiarios.

b) La Comisión Nacional de Arbitraje Médico (CONAMED) creada en 1996 como órgano desconcentrado de la Secretaría de Salud, cuya misión es propiciar las relaciones sanas entre los profesionales de la salud y sus pacientes. Esto a través de la mediación para la resolución de conflictos entre pacientes, sus familiares y personal para la salud (médico y enfermeras, entre otros) en los ámbitos público y privado. Siempre actuando con imparcialidad, objetividad, confidencialidad y respeto mediante la gestión, la conciliación y el arbitraje para ser un factor de éxito y confianza en los servicios de salud de México, a fin de tutelar el Derecho a la Protección de la Salud.

Ambas instancias, a partir de los registros de errores durante la atención médica, emiten recomendaciones para el diseño de métodos, procedimientos o protocolos, pero en especial, para mejorar la práctica de los profesionales de salud del país y la seguridad de los pacientes. ${ }^{10}$

Por su parte, la Comisión Federal para la Protección del riesgo Sanitario (COFEPRIS) juega un papel importante pues ejerce las atribuciones de regulación, control y fomento sanitario en lo relativo al control y vigilancia de los establecimientos de salud, la prevención y el control de los efectos nocivos de los factores ambientales en la salud del hombre. ${ }^{11}$
Es importante señalar que prácticamente en cada Institución de salud existen comités tanto de seguridad como de calidad para los pacientes hospitalizados. ${ }^{12}$

Dada la relevancia del tema, en el Instituto Nacional de Perinatología "Isidro Espinosa de los Reyes" se asignó a un grupo de profesionales la organización, planeación e implementación de aspectos relacionados con la seguridad del paciente. Una de las estrategias de acción es aplicar una metodología rastreadora, la cual consiste en seleccionar un paciente denominado "complejo", el cual debe contar con algunas características distintivas; recibir atención en diferentes servicios del hospital, contar con un promedio mayor de días de estancia hospitalaria y contar con un diagnóstico que concuerde con los de mayor morbilidad y/o mortalidad del hospital. Una vez identificado, se hace una revisión del expediente clínico y se visitan los servicios o áreas donde el paciente recibió atención. Así mismo se realizan cuatro rastreadores en forma de sistemas: Medición, Control de infecciones, Información y Seguridad de las instalaciones. ${ }^{13}$ Lo anterior muestra el reconocimiento y el interés de esta Institución por contribuir a mejorar la seguridad y calidad de la atención.

Con el marco antes señalado y la revisión bibliográfica realizada, se estableció como objetivo la elaboración de esta propuesta de lineamientos de seguridad del paciente encaminadas al cuidado específico del neonato. Se tomó como fundamento las metas establecidas por la OMS sobre seguridad del paciente hospitalizado y los avances institucionales en este campo.
Hablar de seguridad en el paciente neonato implica, entre otras cosas, realizar un análisis y reflexión bioética que involucra a todos los profesionales que brindan la atención a fin de reconocer la importancia del reporte y notificación de eventos adverso

\section{ESTRATEGIAS DE SEGURIDAD} EN EL NEONATO

\section{Identificación correcta del Paciente:}

Implica que el paciente (en este caso el neonato) durante toda su estancia hospitalaria tenga una pulsera de identificación que lleve los datos necesarios (apellidos, número de registro, género, fecha y hora de nacimiento) que corrobore su identidad.

Lo anterior es de suma importancia tal como lo muestra un estudio descriptivo realizado por García y cols. en una unidad de alta especialidad de gineco-pediatría, quienes evaluaron de forma simultánea, la congruencia, legibilidad y datos completos en la tarjeta de identificación de los recién nacidos. Lo realizaron cotejando 100 formas de registro diario, tarjetas de identificación, tiras mecanografiadas, pulseras y expedientes clínicos. Se encontró que la identificación fue realizada en un $72 \%$ pero hubo errores como letra no legible, falta de congruencia de datos e incongruencia entre la tarjeta y la 
pulsera que identificaba al paciente. Al momento de revisar en los recién nacidos las tiras de identificación en tórax, muñeca y tobillo solo un $73 \%$ las portaban. Conclusión: estas medidas deben garantizar calidad en la identificación del neonato asegurando el legítimo derecho a la identidad y privilegio del vínculo materno. ${ }^{14}$

Buscando errores en la identificación de pacientes en la unidad de cuidados intensivos neonatales James E. Gray y cols. concluyeron que ni un solo día estaba libre de riesgo de identificación incorrecta del neonato, pues el número medio de pacientes que se encontraban en situación de riesgo en un día determinado fue de 17 , lo que representa poco más del $50 \%$ del censo diario promedio. Durante todo el año; tiempo en que duró el estudio, el riesgo varió de $20.6 \%$ a un máximo del 72.9\%. Las causas más comunes de errores en la identificación fueron la apariencia similar de días que llevaba hospitalizado el paciente $44 \%$, apellidos idénticos estuvie-

La comunicación entre el personal de salud debe ser tanto escrita como oral de manera completa, oportuna, precisa y comprendida por quien la recibe en relación

a las prescripciones que se aplicarán al neonato. ron presentes en el 34\% y nombres que sonaban parecidos en un $9.7 \%$ de los días, el 26.3\% de los días se mantuvo en riesgo de identificación errónea. Una conclusión del estudio es que en la UCIN a menudo el riesgo en la identificación errónea tiene como fuente la existencia de similitudes, esto persiste incluso después de la exclusión de los nacimientos múltiples y es sustancialmente el más alto que ha sido reportado en otras poblaciones hospitalarias. ${ }^{15}$

A fin de contribuir al cumplimiento de esta medida de seguridad en los neonatos se establecen las siguientes propuestas:

Uso de más de dos identificaciones; el neonato contará con una identificación en brazo derecho y una segunda identificación en pierna izquierda esto con el fin de tener un brazalete seguro en caso de pérdida accidental de alguno de ellos; una tercera identificación será colocada a la madre y la cuarta para el padre permitiendo la identidad con ambos progenitores.

- La identificación deberá contar con folio, registro o código de barras igual al de los padres evitando errores en datos específicos del recién nacido y reconocimiento seguro de los padres.

- Colocar tarjeta con datos generales en cuna; deben registrarse los apellidos de la madre, género, fecha y hora de nacimiento, número de registro hospitalario, servicio asignado y número de cuna. Para la realización de esta tarjeta se debe de verificar si los datos son los correctos.

- No utilizar como identificación el número de cama o servicio; es importante registrar al neonato por su nombre y/o apellidos evitando la confusión con los demás pacientes que se encuentran dentro de la misma área o servicio hospitalario.

- Contar con personal de custodia al momento de ingreso y egreso del recién nacido en los diferentes servicios que corrobore su identidad durante el traslado intra y extrahospitalario.

- Identificación plena del paciente antes de administrar medicamentos, hemoderivados u otros, así como para la realización de exámenes clínicos y de gabinete, verificando que es el neonato correcto.

\section{Mejora de la comunicación efectiva:}

La comunicación entre el personal de salud debe ser tanto escrita como oral de manera completa, oportuna, precisa y comprendida por quien la recibe en relación a las prescripciones que se aplicarán al neonato.

Esta estrategia es muy importante tal como lo muestran Lavalle-Villalobos y cols. en un estudio comparativo realizado en dos fases; el cual giró alrededor del análisis sobre el error médico en la prescripción de medicamentos y el impacto de una intervención educativa. Ellos en la primera fase del estudio determinaron la frecuencia y causa del error en la medicación y en la segunda parte se analizó la respuesta a medidas correctivas. Para ello se revisaron 232 expedientes, $57.3 \%$ tuvieron uno o más errores, de un total de 397 , que corresponde a 2.9 erro- 
res por expediente. En la segunda fase se analizaron 226 expedientes detectando $17.6 \%$ con errores, 111 en total, 2.7 errores por expediente. Concluyeron que las actividades de supervisión y verificación en las indicaciones médicas, apegadas a protocolos de atención médica, rutas críticas y manuales de prescripción pediátrica, influyen en la disminución del error en la prescripción, dándose a conocer un decálogo de seguridad del paciente pediátrico. ${ }^{16}$

A fin de alcanzar esta estrategia las propuestas sugeridas son:

- Verificación de indicaciones terapéuticas por dos personas sea de forma verbal o escrita.

- Escuchar y observar con atención la prescripción terapéutica.

- Repetir la información escuchada, analizando la frase descrita.

- Escritura clara del tratamiento médico y de enfermería; la redacción debe ser completa, precisa y concreta respetando ortografía.

- No utilizar abreviaturas, esto puede traer confusiones y errores de interpretación modificando el cuidado en el neonato.

\section{Mejora en la seguridad de los medicamentos de alto riesgo:}

Deberán estar bien identificados y con algún distintivo los medicamentos considerados de alto riesgo farmacológico. Preferentemente alejados del resto de los demás medicamentos, permitiendo así diferenciarlos para evitar confusión.

Thomas y cols. Revisaron y clasificaron todos los incidentes de seguridad de los pacientes que se encontraban en las Unidades de Cuidados Críticos en Inglaterra y Gales en el año 2008 a través de una agencia de seguridad. Un total de 6649 incidentes se presentaron en 141 organizaciones, teniendo un rango de 23 incidentes por organización; 786 no estaba relacionado con el episodio de cuidados críticos y 248 fueron durante otras etapas de la hospitalización. De los 5615 incidentes restantes, 1726 se produjeron en los primeros momentos del nacimiento, 1298 se asocian con un daño temporal, 15 con daño permanente y 59 asociados a intervenciones necesarias para mantener la vida o pueden estar relacionados con la muerte del paciente. Los principales grupos de incidentes fueron por medicamentos 1450 casos, recursos materiales y humanos en 1289 casos y durante la atención al neonato 1047 casos. Este artículo señala la importancia de dar a conocer el incidente ya que pone de relieve el análisis para mejorar la seguridad del paciente y clasificar así los incidentes. ${ }^{17}$

Por su parte Donoso y cols. reportaron en un estudio de cohorte prospectivo en el área de cuidados críticos que los efectos adversos son comunes y su reporte es posible y útil. La mayoría de ellos se pueden prevenir y su conocimiento es de vital importancia para la seguridad de los pacientes. Este informe comprendió 38 meses de estudio, un total de 2133 niños de los cuales 810 fueron ventilados mecánicamente. Se reportaron 96 eventos adversos ocurridos en 73 pacientes, haciendo un promedio de 2 eventos al mes, el $60 \%$ de ellos ocurrió durante el día en un horario de 8:00 a 21:00 horas. Los even- tos adversos fueron relacionados a ventilación asistida, sin embargo las complicaciones por fármacos superan su impacto. ${ }^{18}$

El factor humano como determinante de la ocurrencia del incidente es muy importante, pero también se han señalado otros aspectos, Donchin y cols. identificaron la falta de estandarización, insuficiente rotulado de los medicamentos, pobre documentación y escasa comunicación, como los principales agentes generadores de éstos. ${ }^{19}$

Considerando que esta estrategia resulta también vital, las propuesta son:

- Preparar medicamentos sin distractores, ya que al dosificar el fármaco es muy fácil equivocarse y cargar dosis incorrectas. Los laboratorios farmacológicos no cuentan con fórmulas dosificadas para el paciente neonato haciendo esto más peligroso si no se aplica la dosis correcta.

- Preparación en área estéril (preferentemente en campana de flujo laminar), lo que garantiza que el medicamento está libre de microorganismos que pueden generar algún proceso infeccioso.

- Preparación y administración de medicamento por la misma persona; lo cual garantiza que el medicamento fue el prescrito y administrado al paciente correcto.

- El uso de catéteres centrales disminuye el número de punciones para la infusión de soluciones y medicamentos por vía endovenosa.

- No administrar 
medicamentos en bolo ya que su concentración genera lesión vascular (flebitis, extravasación, quemadura) o inestabilidad hemodinámica del neonato.

- Uso de bombas de infusión evitando el paso de más volumen del líquido del indicado y se pueden administrar microdosis sin riesgo.

- Vigilar concentración y dosis exacta de medicamentos para no administrar dosis incorrectas y aforar acorde a prescripción del medicamento.

- Utilizar el nombre genérico del medicamento, lo cual evita confusión por el alto número de medicamentos similares en nombre comercial.

- Aplicar los 10 correctos: Medicamento correcto, verificar la fecha de vencimiento del medicamento, paciente correcto, dosis correcta, administrar el medicamento a la hora correcta, vía de administración correcta, preparar usted mismo el medicamento, no administre un medicamento que usted no halla preparado, administrar usted mismo el medicamento, registrar usted mismo el medicamento y la hora de administración en la historia del paciente, tener responsabilidad de la administración del medicamento.

\section{Garantizar cirugías en el} lugar correcto, el procedimiento correcto y el paciente correcto:

Verificar en todo momento del acto quirúrgico (pre y tras operatorio) que se realice el procedimiento quirúrgico en el sitio de intervención programado para el paciente.

Como un ejemplo, en la Universidad de Buenos Aires, Heredia MF, describe la importancia de la cirugía cardiaca segura en caso de ventrículo único (Glenn y Fontan) en el neonato. La circulación extracorpórea en este procedimiento es muy importante, debiendo tener en cuenta diferentes aspectos fisiopatológicos específicos, como la inmadurez de los órganos en neonatos, la circulación cerebral restringida, el volumen de líquido corporal (su distribución) así como la disminución de la temperatura corporal llegando a grados de hipotermia. ${ }^{20}$

La seguridad del neonato durante la cirugía de cualquier tipo es fundamental, derivado de ello las propuestas para alcanzarla son:

- Uso de los tiempos fuera ${ }^{21}$ (sing in, time out, sign out), por parte de todo el personal que participa en la cirugía del neonato corroborando todos los datos acorde a cada momento de la cirugía.

- Monitorización continua de signos vitales permitiendo identificar oportunamente alguna descompensación hemodinámica y en caso necesario actuar sin contratiempos.

- Mantener normotermia, normoxemia, normovolemia y normoglucemia.

- Manejo del dolor aplicando de primera instancia medidas no farmacológicas y posteriormente analgésicos que ayuden a disminuir su intensidad.
- Si en factible, realizar la cirugía en la unidad del paciente evitando traslado de un servicio a otro con el riesgo de complicaciones durante el mismo.

- Ambiente térmico neutro con uso de servocontrol, evitando periodos de hipotermia y distermias que alteren el estado hemodinámico del paciente.

- Uso de bolsa de plástico para evitar pérdidas insensibles.

\section{Reducción de las infecciones adquiridas por la atención:}

Siempre se deberán aplicar medidas estándar por parte de personal de salud que interviene al paciente buscando evitar que éste adquiera alguna infección dentro del hospital.

En Argentina, Barrionuevo y Esandib realizaron un estudio de corte transversal basado en la revisión de historias clínicas neonatales en dos etapas: rastreo a través de indicadores que identifican las historias clínicas con mayor riesgo de ocurrencia de eventos adversos y confirmatorias que hace referencia a la valoración de la existencia, tipo, categoría, prevenibilidad y consecuencias del evento adverso. Encontraron 146 eventos adversos en 82 internaciones $(16,9 \%)$ y un promedio de 3 eventos adversos cada 10 internaciones. De éstos más del $90 \%$ fueron considerados prevenibles. Los más frecuentes fueron: infecciones intrahospitalarias, eventos relacionados con el manejo de catéteres y las extubaciones. La probabilidad de eventos adversos se asoció inversamente con el peso al nacer, edad gestacional y estancia hospitalaria. Como conclusión refieren que la pobla- 
ción neonatal y sobre todo el prematuro, constituye un grupo de gran vulnerabilidad para presentar eventos adversos. La mayoría de éstos eran potencialmente prevenibles, lo cual evidencia la necesidad de implementar intervenciones eficaces que permitan mejorar la seguridad y calidad de atención. ${ }^{22}$

En un estudio de casos y controles en pacientes neonatos del área de cuidados intensivos Gutiérrez y cols, consideraron como factores de riesgo para sepsis neonatal la ruptura prematura de membranas, corioamnioitis, tipo de nacimiento, infecciones de vías urinarias y vaginales en la madre, la multi-invasión con catéteres centrales y arteriales además de la multipunción.

Identificaron que los factores generales de morbimortalidad fueron; hijo de madre con enfermedad sistémica como hipertensión arterial del embarazo y diabetes gestacional, asfixia neonatal moderada o severa, apgar bajo recuperado, trauma obstétrico o bien datos clínicos o de laboratorio que sugieren sepsis. Se incluyeron 62 neonatos,

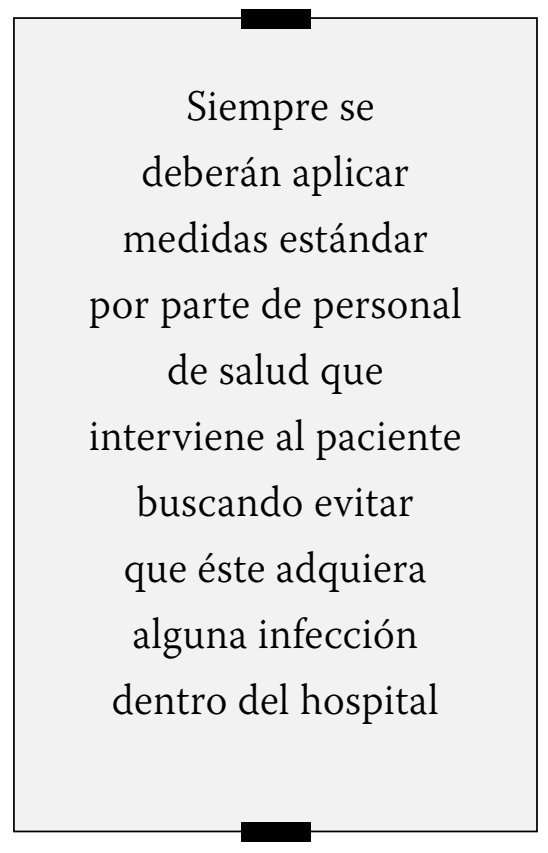

31 pacientes tuvieron sepsis, la vía de nacimiento $76.4 \%$ por operación cesárea y el $23.6 \%$ por vía vaginal, el apgar menor a 7 a los 5 minutos mostró ser un factor de riesgo para la presencia de sepsis neonatal aunque no se explican la razón después de haber hecho una exhaustiva revisión bibliográfica, por lo que puede ser motivo de futura investigación. ${ }^{23}$

Propuestas para alcanzar esta estrategia:

- Lavado de manos en los 5 momentos de atención y uso de gel antibacterial.

- Conocer antecedentes maternos e identificar datos de riesgo de infección, a fin de determinar las posibles causas de complicación(es) en el estado de salud agregada para el neonato.

- Fomentar la alimentación al seno materno evitando el uso de biberón que puede ser un vector en infecciones gastrointestinales.

- Tratamiento certero y correcto de infecciones, mediante un diagnóstico oportuno además de identificar signos y síntomas de alarma para evitar daños a la salud y secuelas a largo plazo que pueden causar modificaciones en la calidad de vida a futuro.

- No canalizar miembros pélvicos por el riesgo de infección por contaminación sobre todo en los neonatos que se encuentran con tratamiento de fototerapia sin colocación de pañal.

- Implementar programa de manejo mínimo, el cual establece las horas contacto, evitando aumento en el número de manipulaciones durante la estancia hospitalaria en donde el personal de enfermería después de realizar una valoración determina el momento y tiempo oportuno para realizar las intervenciones que requiere el neonato.

- Mantener circuito cerrado durante la aspiración de secreciones, efectuar este procedimiento disminuye en gran medida el ingreso de microorganismos a la vía respiratoria.

- Aislamientos de pacientes con padecimientos infectocontagiosos tomando todas las medidas de seguridad para evitar posibles infecciones nosocomiales dentro de las áreas neonatales, en donde la unidad del paciente contará con todo el equipo y material necesario para el cuidado individualizado que éste requiere.

- Respetar circulación de ingreso y egreso en terapias neonatales; el trayecto de aire que entra y sale del servicio de neonatología debe estar controlado evitando así con el movimiento ingresar por medio de partículas aéreas agentes contaminantes.

- Toma periódica de cultivos y limpieza profunda en los servicios hospitalarios que manejen pacientes neonatos, tanto del área física como del personal que brinda de forma directa cuidados.

- Evitar el acceso a personas con proceso(s) infeccioso(s), evitando así posibles contagios que comprometen el sistema 
inmunológico de cada neonato que se encuentre hospitalizado.

- Implementación de Programa Madre Canguro, ${ }^{24}$ el apego con su mamá estimula el sistema inmunológico, además se incrementa en peso del neonato de forma rápida dando pauta a una alta hospitalaria precoz evitando el riesgo de posibles infecciones nosocomiales.

\section{Reducción del riesgo de lesiones al paciente por caídas:}

Resulta fundamental valorar y aplicar acciones en los pacientes con mayor riesgo de presentar una caída así como brindar orientación al familiar.

En la búsqueda de información en diferentes bases de datos no hay referencia de estudios sobre este punto en neonatos, sólo se hace referencia para evaluar algunas medidas preventivas para evitar caídas en neonatos en una hoja de evaluación del Programa de Estímulos y Recompensas por parte de la Secretaría de Salud en México ${ }^{25}$ para el personal de enfermería adscrito en áreas neonatales.

Algunas propuestas alrededor de esta estrategia son:

- Mantener la iluminación del área en un 100\%, permitiendo una observación y vigilancia continua de todos los neonatos dentro del servicio, particularmente en el turno nocturno.

- Los barandales de cuna radiante deberán estar elevados; el movimiento propio del neonato que busca algún sitio de contención puede generar alguna caída.
- Cerrar las ventanas de las incubadoras posterior a su asistencia y de la visita de los padres, corroborando periódicamente que se mantengan así.

- Reunir todo el material necesario antes de realizar algún procedimiento con la finalidad de no dejar ningún momento solo al neonato y estar vigilando constantemente.

- Capacitar al familiar sobre la importancia de seguir las medidas de seguridad y prevención de caídas al momento de cargarlo. Es importante tomar en cuenta las condiciones maternas y su disposición para el contacto con su hijo. La enfermera deberá de estar pendiente de la respuesta del binomio al contacto mutuo.

- Colocar rollos o nidos para dar contención evitando el desplazamiento. Esto ayuda a que el neonato sienta límites para desplazarse y permanezca más tiempo tranquilo y sin movimientos bruscos que puedan originar una caída.

- Traslado intra y/o extrahospitalario obligadamente en incubadora equipada, dejando al neonato seguro en su destino. Esta medida permite monitorizar al paciente de forma continua, así como evitar periodos de hipotermia, hipoxia y riesgos de caídas por llevarlo en brazos.

\section{Conclusiones}

Aplicar los lineamientos específicos de seguridad en el neonato hospi- talizado debe ser una prioridad del profesional de enfermería con el fin de garantizar una cultura de calidad en la atención. Omitir alguno de ellos lo expondrá a riesgos innecesarios que pueden originar eventos adversos, situación que afectaría al paciente y a su familia.

Es de suma importancia que las Instituciones en salud que atienden neonatos registren y den a conocer los eventos adversos y las estrategias utilizadas para garantizar la seguridad en este grupo de pacientes durante su estancia hospitalaria, con el fin de dar continuidad a los programas establecidos dentro de cada área neonatal de los distintos hospitales y dar a conocer los avances específicos en este tema.

\section{Referencias Bibliográficas}

1. Organización Mundial de la Salud.

Nueve soluciones para la seguridad del paciente. Washington/Ginebra, Mayo 2007. [Consulta 20 de enero 2012]. Disponible en: http:// bit.ly/x6EtC8

2 Samra HA, McGrath JM, Rollins W. Patient Safety in the NICU: a comprehensive review. J Perinat Neonat Nursing 2011; 25 (2): 123-132. Disponible en: http://bit. ly/lUU5kU

3 Suresh GK. Measuring Patient Safety in Neonatology. Am J Perinatol. 2012; 29(1):19-26. Disponible en: http://bit.ly/NmoeAe

4. Ramírez R. Catalogación del Recién Nacido. En: Avila-Rojas A y cols. Servicio Neonatología Hospital Clínico Universidad de Chile. 2001. Pp 9-17 [Consulta 13 de enero 2012] Disponible en: http:// bit.ly/QbcVeA

5 Montserrat D. Taller regional Sistemas de Notificación. OMS/OPS/ CONAMED, México, D.F. 12-13 
agosto 2010. Disponible en: http:// bit.ly/T1hsx3

6 Ursprung R, Gray JE, Edwards WH, et al. Real time patient safety audits: improving safety every day. Qual Saf Health Care 2005 14 (4): 284-289 Disponible en: http://1. usa.gov/RIqf6g

7 Villareal E. Seguridad de los pacientes, un compromiso de todos para un cuidado de calidad. Salud Uninorte 2007; 23 (1): 112-119. Disponible en: http://bit.ly/RDKgQR

8 Kosier B, Erb G, Olivieri R. Enfermería Fundamental: conceptos, procesos y práctica. Madrid: McGraw-Hill. Interamericana, 4ta edición, tomo I, 1993.

9 Aguirre-Gas H. Conferencia sobre "Seguridad del paciente". 2da. Reunión sobre calidad en la atención, IMSS, Ixtapa-Zihuatanejo, Gro. Mex. 6 de abril de 2006. [Consulta 16 de febrero 2012] Disponible en http://bit.ly/jjMvY3

10 Presidencia de la República. Decreto de creación de la Comisión $\mathrm{Na}$ cional de Arbitraje Médico. México: Diario Oficial de la Federación 3-06-1996. Disponible en: http:// bit.ly/Vhmg7B

11 SSA. Atribuciones, funciones y características de Cofepris. México: Gobierno Federal. Salud.Cofepris. Disponible en: http://bit.ly/LP68r6

12 Campos-Castolo M, Carrillo-Jaimes A. Reporte de errores médicos como estrategia para la prevención de eventos adversos. Revista CONAMED 2008; 13 (2): 17-22. [Consultado 15 de enero 2012] Disponible en: http://bit.ly/ Qhztb1

13 Instituto Nacional de Perinatología. Departamento de Estadística y Metas institucionales, Dirección de Planeación. México, 2011. [Consultado 15 de enero 2012]
Disponible en: http://www.inper. edu.mx/

14 García-Salazar RM, Gómez-Flores

L, Cintora-Bermúdez L, JiménezLópez GN, Martínez-Torreblanca O, Montesinos-Sánchez IP. Apego a las medidas de seguridad en el procedimiento de identificación inequívoca del recién nacido. Rev Enferm Inst Mex Seguro Soc 2009; 17 (3): 129-132. Disponible en http://bit.ly/UI0J5k

15 Gray JE, Suresh G, Ursprung R, Edwards WH, Nickerson J, Shiono PH, y cols. Patient Misidentification in the Neonatal Intensive Care Unit: Quantification of Risk. Pediatrics 2006; 117; e43. [Consulta 21 de enero 2012]Disponible en: http://bit.ly/THnOlV

16 Lavalle-Villalobos A, Payro-Cheng TJ, Martínez-Cervantes KA, Torres Narváez P, Hernández-Delgado L, Flores-Nava G. El error médico en la prescripción de medicamentos y el impacto de una intervención educativa. [Consulta 2 marzo 2012] Disponible en: http:// bit.ly/RJ36k8

17 Thomas AN, Panchagnula U, Taylor RJ. Review of patient safety incidents submitted from Critical Care Units in England \& Wales to the UK National Patient Safety Agency. Anaesthesia 2009, 64(11): 1178-85. [Consulta 18 de enero 2012]Disponible en: http://bit.ly/ QbvLC7

18 Donoso-Fuentes A, Fuentes I. Eventos adversos en la UCI. Rev. Chilena de Pediatría. 2004; 75(3): 233-39. [Consulta 18 de enero 2012] Disponible en: http://bit.ly/ SCOH1G

19 Donchin Y, et al. A look into the nature and causes of human errors in the intensive care unit. Crit Care Med. 1995 Feb;23(2):294-300.
20 Heredia MF. Ventrículo Único: Cirugía de Glenn y Fontan. Rev Latinoamer Tecnol Extracorp 2007; X1V (2): 7-25. [Consulta 14 de Febrero 2012], Disponible en http:// bit.ly/RJar32

21 World Alliance for patient safety. WHO surgical safety checklist and implementation manual. USA: WHO, 2008. Disponible en: bit.ly/ MEkKCV

22 Barrionuevo LS, Esandib ME. Epidemiología de eventos adversos en el servicio de neonatología de un hospital público regional en la Argentina. Arch Argent Pediatr 2010;108(4):303-310. [Consulta 25 de enero 2012], Disponible en: http://bit.ly/P9lnZz

23 Gutiérrez-Muñoz VH, GutiérrezMuñoz J, Rosas-Barrientos V. Factores de riesgo en sepsis neonatal en un hospital de tercer nivel en la ciudad de México. Revista de Especialidades Médico Quirúrgicas 2005; 10(2): 21-24. [Consulta 25 de enero 2012], Disponible en: http:// bit.ly/QbA7cs

24 Torres, J. Palencia,D. Sánchez, DM. García,J. Rey H. Echandía,CA. Programa Madre Canguro: primeros resultados de una cohorte de niños seguidos desde la unidad neonatal hasta la semana 40 de edad postconcepcional. Colombia Médica Vol. 37 № 2, 2006 (AbrilJunio). 96-101, Disponible en: http://bit.ly/RBmWS9

25 Comité Nacional de Estímulos a la Calidad del Desempeño del Personal de Salud. Evaluación de Enfermería en el área de Neonatos. México, SSA. [Consulta 3 de enero 2012], Disponible en: http://bit. ly/Scwr6T 